\title{
Disseminated peritoneal leiomyomatosis: an unusual presentation of intra-abdominal lesion mimicking disseminated malignancy
}

Sweta Soni, Puneet Pareek, Satya Narayan

Department of Radiation Oncology, All India Institute of Medical Sciences, Jodhpur, Rajasthan, India

DOI: $10.15386 / \mathrm{mpr}-1421$

Manuscript received: 17.07.2019

Received in revised form: 04.09.2019 Accepted: 05.10.2019

Address for correspondence: sonisweta@hotmail.com

This work is licensed under a Creative Commons Attribution-NonCommercialNoDerivatives 4.0 International License

\begin{abstract}
Leiomyoma are commonly seen as benign smooth muscle tumors of the uterus. Smooth muscle tumors with unusual growth pattern are rare and include 3 primary neoplasms: intravenous leiomyomatosis (IVL), benign metastasizing leiomyoma (BML) and disseminated peritoneal leiomyomatosis (DPL). DPL is a rare benign disease, often giving the appearance of metastatic ovarian or peritoneal carcinoma. It is a disease that predominately affects women at their reproductive age. The risk of malignant transformation is $2-5 \%$. There are no standard treatment guidelines for the management of the DPL. The unusual presentation of the disease delays the diagnosis or is often misdiagnosed and thus over treated, which may lead to increased morbidity and mortality. Therefore careful consideration and high index of suspicion is required for the proper management of such cases.
\end{abstract}

Keywords: leiomyoma, peritoneal leiomyomatosis, uterine neoplasm, benign, DPL

\section{Introduction}

Leiomyomas represent the most common gynecologic and uterine neoplasms. $20 \%-30 \%$ of women older than 35 years have uterine leiomyomas that present clinically. These lesions include a range of presentations and extensions, from within the uterus to anywhere in the body. Disseminated peritoneal leiomyomatosis is a rare benign diseases of unknown etiology of women in the reproductive age group mimicking disseminated malignancy. Unusual growth patterns or unusual locations make their identification more challenging both clinically and radiologically. Therefore in this article, we will discuss about clinico-pathologic characteristic, radiological features and prognosis of this rare type of diseases.

\section{Case presentation}

A 38-year-old female, unmarried, with regular menstrual history, no comorbidities, no addiction, presented with complaints of abdominal discomfort on and off. She had past history of surgery for uterine fibroids in 2010. She had no family history of any malignancy (like ovary, breast, others). Also she had no history of use of any oral contraceptive pills. She was investigated and abdomen ultrasound was suggestive of mild hepatomegaly, bulky uterus with intramural fibroid in anterior wall of the body of uterus. Bilateral adnexal masses of variable size were seen and bilateral ovaries were not identified. CT (computed tomography) of the thorax and abdomen (Figure 1) was suggestive of multiple well defined lobulated solid masses in the abdomen, pelvis and peritoneal cavity in bilateral iliac fossa, left lumbar region and pouch of Douglas (approximately 10 in number), largest $6 \times 6.6 \mathrm{~cm}$ in left lumbar region. Disseminated peritoneal leiomyomatosis. Uterus showed illdefined enhancing lesion $4 \times 3.2 \mathrm{~cm}$ anterior wall of myometrium. Fibroid. Lung and liver study was normal. CA 125: $151.9 \mathrm{U} / \mathrm{mL}$, AFP (Alpha fetoprotein): $2.54 \mathrm{ng} / \mathrm{mL}$, Beta HCG (Human chorionic gonadotropin): $<1.2 \mathrm{mlU} / \mathrm{mL}$. PET CT (Positron Emission Tomography 
- Computed Tomography) Scan (Figure 1) was suggestive of low grade FDG (fluorodeoxyglucose)-avid, well defined enhancing lesion with lobulated margins, enhancing the lesion seen in the anterior myometrium measuring $34 \times 31$ with SUV max 3.3. Moderate grade FDG (Standard Uptake Value, SUV 5) avid lesion is seen in the fundus measuring $10 \mathrm{~mm}$. Low to moderate FDG avid similar enhancing lobulated lesion are seen in both ovaries, along both adnexa and peritoneum, largest left iliac fossa $86 \times 61 \times 57 \mathrm{~mm}$ in size (SUV 4.3). Right and left adnexal lesion measured $44 \times 35 \times 30 \mathrm{~mm}$ and $44 \times 42 \times 27 \mathrm{~mm}$ respectively.

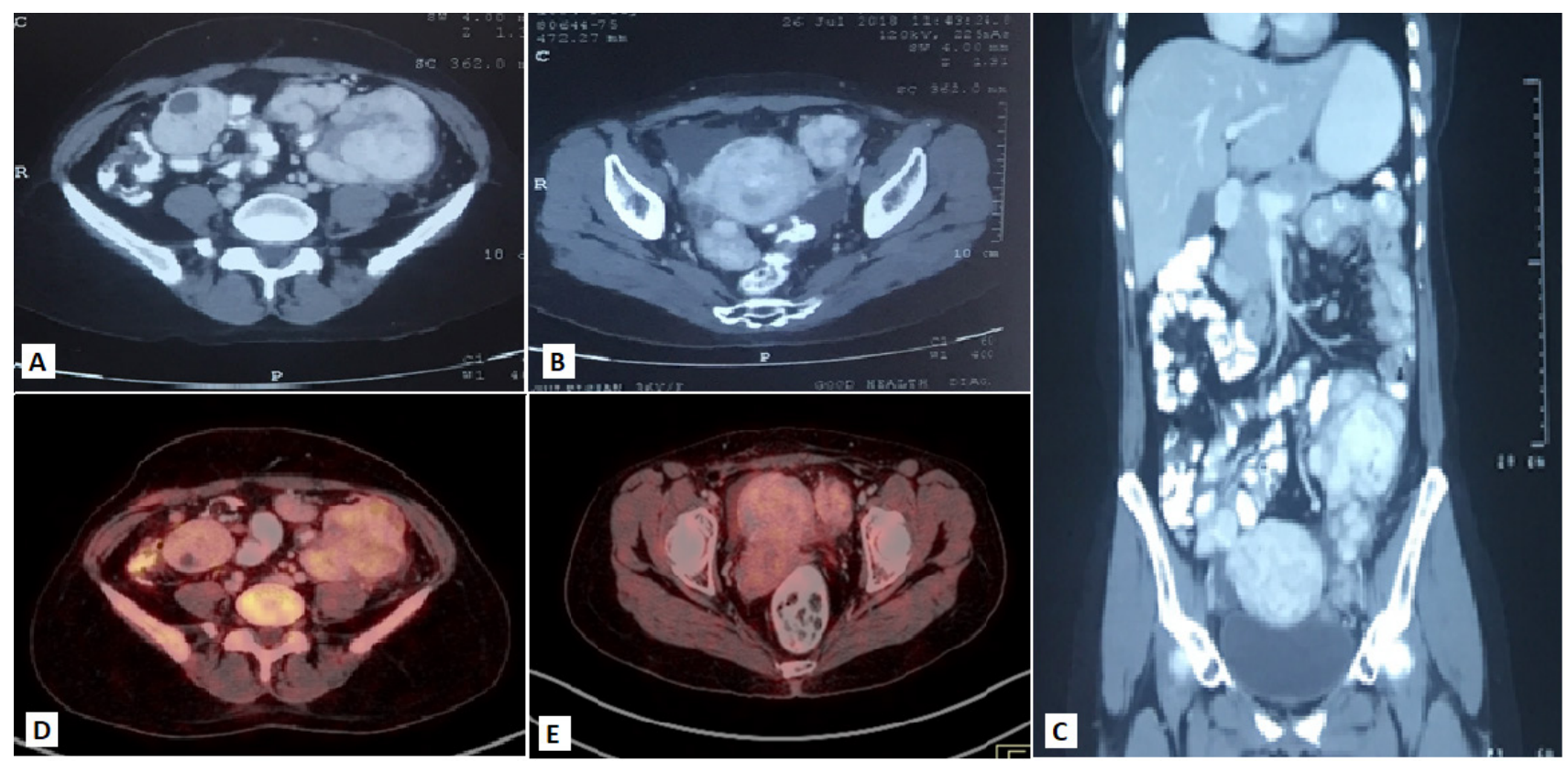

Figure 1. CT thorax and abdomen images suggestive of multiple well defined lobulated solid mass in abdomen, pelvis, peritoneal cavity, in bilateral iliac fossa and left lumbar region with uterine fibroid (A,B,C). PET CT Scan images suggestive of low grade FDG avid disseminated peritoneal nodule with uterine fibroid $(\mathrm{D}, \mathrm{E})$.

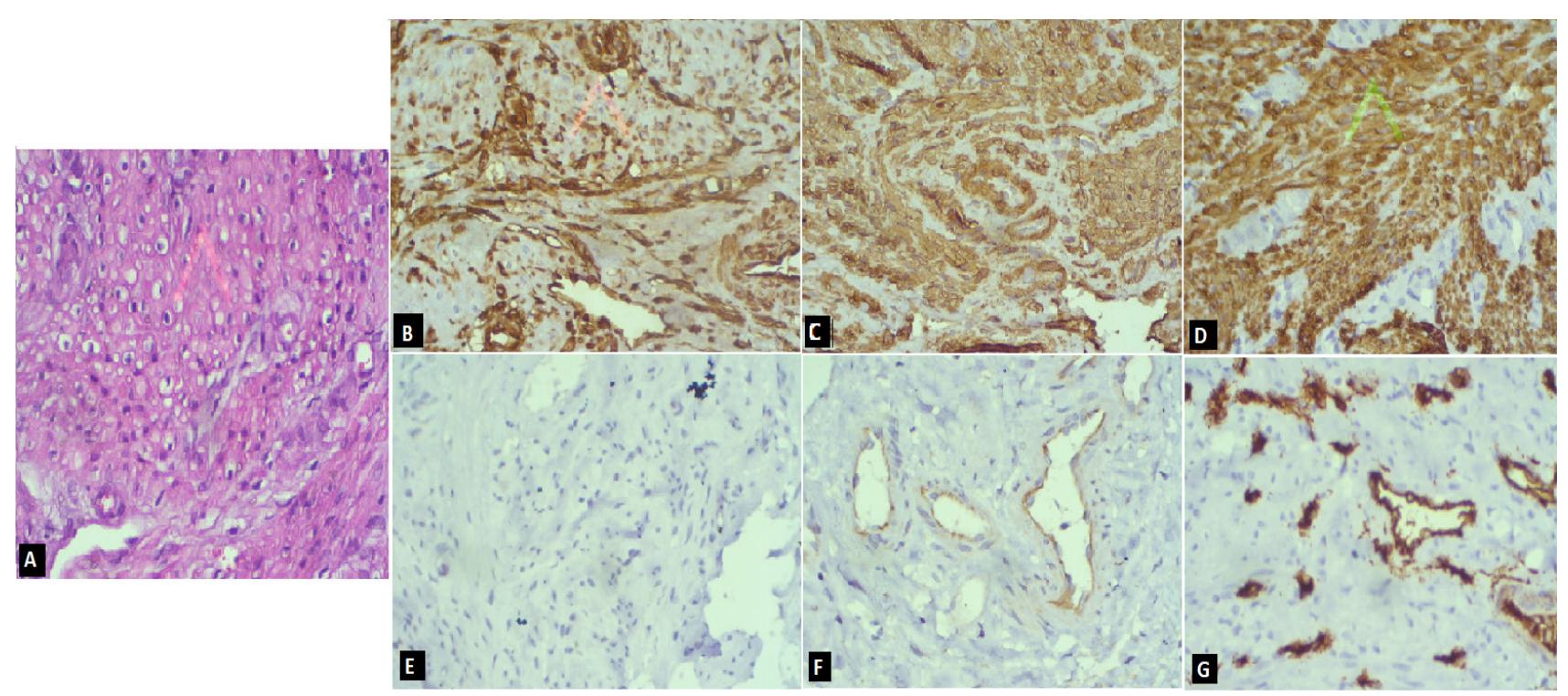

Figure 2. (A) Histological examination of peritoneal nodule shows fascicle and bundles of spindle cell with many small capillaries. No significant atypia / mitosis/ necrosis seen (H \& E). The immunohistochemical evaluation is positive for (B) Vimentin (C) Smooth muscle actin (D) Desmin and negative for (E) S 100 (F) PAN CK (G) CD 34. 
Final impression was given as low grade FDG avid disseminated peritoneal nodule with uterine fibroid less likely to be malignant and with no evidence of metabolically active systemic disease or metastasis. Tru-cut biopsy form left lumbar mass was suggestive of low grade spindle cell lesion favoring neural origin. Spindle cells were immunoreactive for vimentin, desmin, SMA (Smooth Muscle Antibody) and negative for CK (cytokeratin), S-100 and CD-34 suggestive of leiomyoma (Figure 2). Patient was started on symptomatic medication like NSAID's (Nonsteroidal anti-inflammatory agents) for pain management. No surgery or any hormonal therapy was attempted, patient was kept on observation and at 6 months of follow up patient was well with no significant complaints apart from occasional abdominal pain which was relived with NSAID's.

\section{Discussion}

Leiomyoma are smooth muscle tumors that are common to the uterus, but uterine smooth muscle tumor with unusual growth pattern are rare and include 3 primary neoplasms: intravenous leiomyomatosis (IVL), benign metastasizing leiomyoma (BML) and disseminated peritoneal leiomyomatosis (DPL) [1]. However, these atypical locations of these tumors present a diagnostic dilemma regarding their origin and benign nature.

Disseminated Peritoneal Leiomyomatosis (DPL) is a rare benign disease, often giving the appearance of metastatic ovarian or peritoneal carcinoma. The estimated prevalence of DPL is $<1 / 10,00,000$ with fewer than 150 cases reported in literature to this date [2]. Such a case was first described by Willson and Peale in 1952 [3]. It is suspected that this disease originates from a metaplasia of submesothelial multipotent mesenchymal cells. Although it's not clear if the stimulus to smooth cell differentiation is hormonal, genetic or both [4,5]. Also, it could be induced after morcellation of myoma during laproscopy [6]. The disease is generally associated with high levels of exogenous or endogenous female gonadal steroids [7].

Disseminated Peritoneal Leiomyomatosis (DPL) is a disease that predominately affects women in the reproductive age group. Very few cases have been described in post-menopausal women and in few men [8]. Although this disease is benign, the risk of malignant transformation is $2-5 \%$, as published in one of the studies [9].

Imaging plays an important role in differentiating this primarily benign condition with other ominous pathologies of the peritoneum. On ultrasound examination, these are seen as well-defined homogeneously hypoechoic nodules with a smooth surface. On MRI (Magnetic Resonance Imaging), these appear as hypointense in T1Weighted, and similarly hypo, iso, or hyper intense on T2 Weighted depending on the water content of the tumor [10]. On postcontrast images, they reveal a homogenous enhancement.
MDCT (Multi-Detector Computed Tomography) shows these lesions as hypodense, well-circumscribed solid nodules with homogenous enhancement. The adjacent peritoneum is usually normal without any thickening or abnormal enhancement. The chief imaging differential diagnoses for DPL are peritoneal carcinomatosis, disseminated leiomyosarcoma, and endometriosis [5].

The most common feature of peritoneal carcinomatosis is ascites, peritoneal thickening with increased enhancement, peritoneal nodules, omental nodules and caking, thickening of the bowel wall, and adenopathy. Disseminated leiomyosarcoma presents as peritoneal nodules. But DPL is isometabolic in PET-CT, leiomyosarcomas are hypermetabolic [11].

Typically, the macroscopic appearance of DPL is the presence of multiple hard rubbery nodules studded diffusely over the peritoneal surfaces, primarily in the lower or middle abdomen and pelvis. Microscopic examination shows subperitoneal nodules with typical interlacing of smoothmuscle cells arranged in whorled pattern without atypia, stromal invasion and with no or low mitotic activity [1].

No standard treatment guidelines exist for the management of the DPL. Treatment needs individualization taking into consideration the patient's age, desire for conception and symptomatology. Asymptomatic DPL requires no therapy because it is usually impossible to remove all nodules and the disease has a benign clinically indolent course. Treatment is necessary in patients with symptomatic disease and in those with growing or recurrent lesions. Because regression has been described with declining levels of estrogen (i.e., menopause), bilateral salpingooophorectomy or GnRh (Gonadotropin Releasing Hormone) analogue therapy should be considered before contemplating surgical excision of the metastatic nodules. Surgical excision of the metastatic nodules is necessary in the rare patient with large symptomatic lesions that are refractory to hormonal therapy or in patients who refuse surgical castration $[1,2,9,12]$. Hysterectomy per se is not beneficial but is considered in patients with associated uterine symptoms [13]. In post-menopausal woman aromatase inhibitor has been used with success to treat non resectable tumors [14].

Studies have shown that disseminated peritoneal leiomyomatosis (DPL) usually has fair prognosis. However, in some cases it may turn fatal.

\section{Conclusion}

Disseminated peritoneal leiomyomatosis is a rare benign disease mimicking disseminated malignancy. Because of rarity and possible unfamiliarity it is often misdiagnosed and thus over treated and on many occasions extensive dissections are performed which may lead to increased morbidity and mortality. therefore careful consideration and high index of suspicion is required for the proper management of such cases. 


\section{References}

1. Vaquero ME, Magrina JF, Leslie KO. Uterine smooth-muscle tumors with unusual growth patterns. J Minim Invasive Gynecol. 2009;16:263-268.

2. Saha PK, Dhaliwal LK, Gainder S, Bagga R, Joshi B, Saha $\mathrm{SC}$, et al. Disseminated peritoneal leiomyomatosis: a rare entity with diagnostic conundrum. Int J Reprod Contracept Obstet Gynecol. 2015;4:1194-1196.

3. Willson JR, Peale AR. Multiple peritoneal leiomyomas associated with a granulosa-cell tumor of the ovary. Am J Obstet Gynecol. 1952;64:204-208.

4. Al-Talib A, Tulandi T. Pathophysiology and possible iatrogenic cause of leiomyomatosis peritonealis disseminata. Gynecol Obstet Invest. 2010;69:239-244.

5. Fasih N, Prasad Shanbhogue AK, Macdonald DB, FraserHill MA, Papadatos D, Kielar AZ, et al. Leiomyomas beyond the uterus: unusual locations, rare manifestations. Radiographics. 2008;28:1931-1948.

6. Sekulic M, Moench L, Movahedi-Lankarani S. Disseminated peritoneal leiomyomatosis postmorcellated resection of uterine leiomyomatous tissue. APMIS. 2016;124:1063-1071.

7. Ramesh L, Edozien L. Not all disseminated intra-abdominal lesions are malignant: a case of leiomyomatosis peritonealis disseminata. J Obstet Gynaecol. 2005;25:409.

8. Paul PG, Naik S. Disseminated leiomyomatosis peritonei. Incidental finding in laparoscopy: a case report. Surg Laparosc Endosc PercutanTech. 2010;20:e123-e124.

9. Surmacki P, Sporny S, Tosiak A, Lasota J. Disseminated peritoneal leiomyomatosis coexisting with leiomyoma of the uterine body. Arch Gynecol Obstet. 2006; 273:301-303.

10. Seidman MA, Oduyebo T, Muto MG, Crum CP, Nucci MR, Quade BJ. Peritoneal dissemination complicating morcellation of uterine mesenchymal neoplasms. PLoS One. 2012;7:e50058.

11. Venkataramanan R, Arunachalam P, Arunachalam V, Venkat P. Peritoneal leiomyomatosis - A rare case report. Apollo Medicine. 2015;12:152-154.

12. Düe W, Pickartz H. Immunohistologic detection of estrogen and progesterone receptors in disseminated peritoneal leiomyomatosis. Int J Gynecol Pathol. 1989;8:46-53.

13. Solomon LA, Schimp VL, Ali-Fehmi R, Diamond MP, Munkarah AR. Clinical update of smooth muscle tumors of the uterus. J Minim Invasive Gynecol. 2005;12:401-408.

14. Raspagliesi F, Quattrone P, Grosso G, Cobellis L, di Re E. Malignant degeneration in leiomyomatosis peritonealis disseminata. Gynecol Oncol. 1996;61:272-274. 